Research Article

\title{
Application of KLE-PEM for Random Dynamic Analysis of Nonlinear Train-Track-Bridge System
}

\author{
Lizhong Jiang $\mathbb{D}^{1},{ }^{1}$ Xiang Liu $\mathbb{D},{ }^{1}$ Tuo Zhou $\mathbb{D}^{1},{ }^{1}$ Ping Xiang $\mathbb{D}^{1},{ }^{1}$ Yuanjun Chen $\mathbb{D},{ }^{1}$ \\ Yulin Feng $\mathbb{B}^{2},{ }^{2}$ Zhipeng Lai $\mathbb{B}^{1},{ }^{1}$ and Shanshan Cao $\mathbb{1}^{3}$ \\ ${ }^{1}$ School of Civil Engineering, Central South University, Changsha 410075, Hunan, China \\ ${ }^{2}$ School of Civil Engineering and Architecture, East China Jiaotong University, Nanchang 330013, China \\ ${ }^{3}$ Guangdong Transportation Technology Testing Co., Ltd, Guangzhou 510550, China
}

Correspondence should be addressed to Xiang Liu; liuxiang2017@csu.edu.cn

Received 27 September 2020; Revised 1 December 2020; Accepted 17 December 2020; Published 28 December 2020

Academic Editor: Marcello Vanali

Copyright $(2020$ Lizhong Jiang et al. This is an open access article distributed under the Creative Commons Attribution License, which permits unrestricted use, distribution, and reproduction in any medium, provided the original work is properly cited.

A nonlinear train-track-bridge system (TTBS) considering the random track irregularity and mass of train is discussed. Based on the Karhunen-Loéve theory, the track irregularity is expressed and input into the TTBS, and the result of random response is calculated using the point estimation method. Two cases are used to compare and validate the applicability of the proposed method, which show that the proposed method has a high precision and efficiency. Then, taking a 7-span bridge and a high-speed train as an example, the calculation results of random response of the nonlinear and linear wheel-rail model are compared, and the results show that for the bridge and rail response, the nonlinear and linear models are almost the same. Finally, comparing the calculated probability distribution results with the test results, it shows that the method can be applied to the prediction of actual response range.

\section{Introduction}

Bridge structure occupies a large proportion in high-speed railway (HSR) lines $[1,2]$. Because of inevitable errors in processing, pavement technology, environment, and other potential problems of track structure, random track irregularity occurs in a train-track-bridge system (TTBS) under excitation, affecting the stability and safety of train operation $[3,4]$. When a train passes over a bridge, the bridge is affected by train vibration; simultaneously, the bridge vibration affects the train vibration [5-7]. The interaction between train and bridge vibrations leads to a coupling vibration effect; in this process, the effects on system vibration due to track irregularity cannot be ignored. Various rail irregularities are superimposed by random irregularities of different wavelengths, phases, and amplitudes [8]. This is a complex random process related to the railway line. Therefore, in dynamics analysis, a TTBS should be regarded as a random system. The track irregularity can be expressed by many methods including power spectrum statistics, Karhunen-Loéve expansion (KLE) [9], and others.

In recent years, the random dynamics of the TTBS has attracted much attention. The most common calculation method for random dynamics is Monte Carlo simulation (MCS) $[10,11]$. MCS requires abundant samples to obtain a convergence result in complex engineering problems, indicating that MCS is subjected to many limitations in practical applications. To obtain an efficient and accurate calculation method for the random dynamics of the TTBS, many studies have been conducted. For instance, the pseudo-excitation method was used for the random dynamics analysis of the train-track system and TTBS [12-16]. It is convenient to use the pseudo-excitation method for response power spectrum calculation in both nonstationary and stationary random excitation structural analysis with a high application value. The probability density evolution method (PDEM) was successfully applied in the analysis of train-track and trainbridge coupling dynamics considering track irregularity of randomness [17-21]. An efficient train-bridge random 
response method capable of global sensitivity analysis has been proposed in reference [22]. Wu and Law [23] used the stochastic finite element spectral method and regarded road irregularity as a random process. Jiang [24] used the point estimation method (PEM) to the stochastic TTBS and analyzed the sensitivity of random parameters. The PEM is a highly accurate and fast statistical moment calculation method, first proposed by Rosenblueth [25]. The core of the PEM is the use of the Gaussian integral method to calculate statistical moments.

This paper is a further discussion of the previous works [24, 26, 27]. Hertz contact theory has been used for the calculation of normal contact force between a wheel and rail; the relationship between force and wheel/rail displacement is nonlinear. Under overloading and highspeed operation, strong wheel-rail dynamic effect results in the disruption of vertical dynamic load between the wheel and rail, severely affecting the bridge safety and passenger comfort as well as directly affecting the dynamic response of the bridge [28]. In the TTBS, the treatment of wheel-rail contact force as a nonlinear interaction can more truly reflect the dynamic situation; in addition, in some extreme track irregularity situations such as earthquake [29,30], settlement [31,32], creep, and rail damage $[33,34]$, the wheel may jump from the rail; i.e., the always-close wheel/rail assumption is not suitable for use. These pose a challenge to the application of random vibration theory. Therefore, this paper proposes a nonlinear random dynamics calculation method suitable for the TTBS with stochastic track irregularity. In this method, the track irregularity is expressed by KLE, and then the system response is calculated using the PEM according to the characteristics of KLE. The difference between nonlinear and linear rail bridge systems is discussed as well.

\section{Dynamic Model of Nonlinear TTBS}

2.1. Wheel-Rail Contact. This study focused on the vertical movement of the TTBS; thus, only the vertical relationship of wheel-rail contact is considered. The normal force is the vertical wheel-rail force in this system, which is determined by compression and calculated using the famous Hertz nonlinear elastic contact theory, as follows:

$$
F_{c i}= \begin{cases}\left|\frac{1}{G}\left(z_{w i}-z_{r i}-z_{\text {Roui }}\right)\right|^{1.5}, & z_{w i}-z_{r i}-z_{\text {Roui }} \leq 0, \\ 0, & z_{w i}-z_{r i}-z_{\text {Roui }}>0\end{cases}
$$

where $z_{w i}$ is the vertical displacement of the $i^{\text {th }}$ wheel set; $z_{r i}$ is the vertical displacement of rail at the position of $i^{\text {th }}$ wheel set; $z_{\text {Roui }}$ is the track irregularity at the position of $i^{\text {th }}$ wheelset; and $G$ is the contact constant. For the abrasion type tread, $G=3.86 R^{-0.115} \times 10^{-8}\left(\mathrm{~m} / \mathrm{N}^{2 / 3}\right)$, and $R$ is the radius of wheel rolling circle.

Sometimes, the Hertz spring stiffness can be linearized as follows [35]:

$$
F_{c i}= \begin{cases}-k_{H L}\left|\left(z_{w i}-z_{r i}-z_{\text {Roui }}\right)\right|, & z_{w i}-z_{r i}-z_{\text {Roui }} \leq 0, \\ 0, & z_{w i}-z_{r i}-z_{\text {Roui }}>0,\end{cases}
$$

where $k_{H L}$ is the linearized contact stiffness of wheel-rail and can be calculated as follows:

$$
k_{H L}=\frac{2}{3 G} p_{0}^{(1 / 3)}
$$

where $p_{0}$ is the static load of the wheel.

2.2. Dynamic Model of Train-Track-Bridge. As shown in Figure 1, one carriage consists of one carbody, two bogies, and four wheelsets, which is a multifreedom mass-springdashpot system. In the model, $m_{c}, m_{t}$, and $m_{w}$ are the mass of carbody, bogie, and wheelset, respectively; $J_{\mathcal{o}} J_{t}$, and $J_{w}$ are the moment of inertia of carbody, bogie, and wheelset, respectively; $k_{1}$ and $k_{2}$ are the stiffness of primary and second suspension spring, respectively; and $c_{1}$ and $c_{2}$ are the damping coefficient of primary and second suspension spring, respectively. Each carbody has two degrees of freedom (DOF) $\left(z_{c}, \varphi_{c}\right)$, each bogie has two DOFs $\left(z_{t}, \varphi_{t}\right)$ as well, and each wheel only has one DOF $\left(z_{w}\right)$. Thus, each carriage has 10 DOFs. A dynamic equation of the train can be derived from the energy principle, as follows [36]:

$$
\mathbf{M}_{v} \ddot{\mathbf{X}}_{v}+\mathbf{C}_{v} \dot{\mathbf{X}}_{v}+\mathbf{K}_{v} \mathbf{X}_{v}=\mathbf{F}_{v} .
$$

Elastic Bernoulli-Euler beams are utilized for rail, track slab, and bridge. Two steel rails are equivalent to one. The fasteners between the rail and rail plate are modeled as spring-dashpot systems, and the mortar layer between the track slab and girder is modeled as a continuous springdamping system. The mass of fastener and CA mortar layer is neglected, and the structural damping of the rail and track slab is not considered. The damping of girders is assumed as Rayleigh damping. The entire track-bridge system can be modeled as a three-layer beam, the model can be established by FEM, and the dynamic equation can be written as follows:

$$
\mathbf{M}_{b} \ddot{\mathbf{X}}_{b}+\mathbf{C}_{b} \dot{\mathbf{X}}_{b}+\mathbf{K}_{b} \mathbf{X}_{b}=\mathbf{F}_{b}
$$

where $\mathbf{X}_{b}, \dot{X}_{b}$, and $\ddot{\mathbf{X}}_{b}$ are the displacement, velocity, and acceleration of the bridge and $\mathbf{F}_{b}$ is the force vector of the bridge. The gravity of structure is neglected in $\mathbf{F}_{b}$.

The train and track-bridge system can be coupled using the wheel-rail relationship, and the system dynamic equation can be derived from equations (4) and (5), which can be written as follows:

$$
\begin{aligned}
& {\left[\begin{array}{ll}
\mathbf{M}_{v} & \\
& \mathbf{M}_{b}
\end{array}\right]\left\{\begin{array}{l}
\ddot{\mathbf{X}}_{v} \\
\ddot{\mathbf{X}}_{b}
\end{array}\right\}+\left[\begin{array}{ll}
\mathbf{C}_{v} & \\
& \mathbf{C}_{b}
\end{array}\right]\left\{\begin{array}{l}
\dot{\mathbf{X}}_{v} \\
\dot{\mathbf{X}}_{b}
\end{array}\right\}+\left[\begin{array}{ll}
\mathbf{K}_{v} & \\
& \mathbf{K}_{b}
\end{array}\right]\left\{\begin{array}{l}
\mathbf{X}_{v} \\
\mathbf{X}_{b}
\end{array}\right\}} \\
& \quad=\left\{\begin{array}{l}
\mathbf{F}_{v} \\
\mathbf{F}_{b}
\end{array}\right\} .
\end{aligned}
$$

The numerical time step-by-step integral method can be used to solve the system dynamic equation. Equation (1) or 


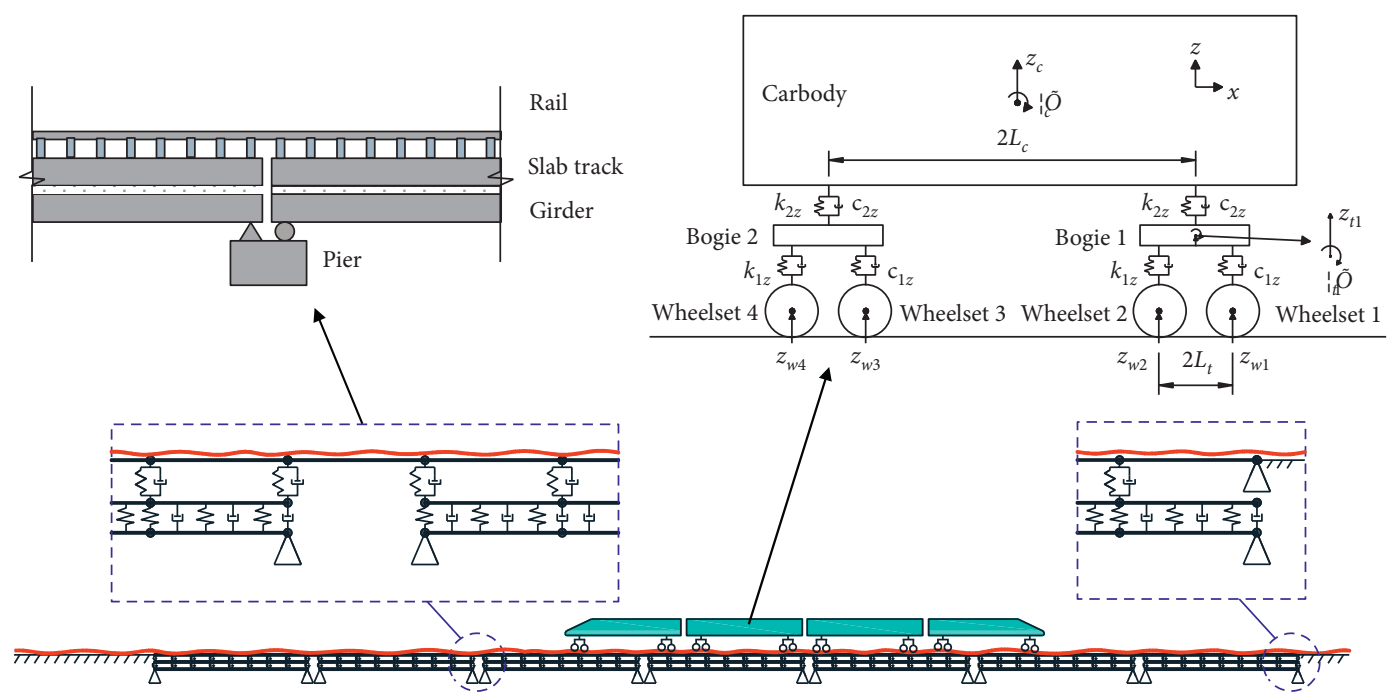

Figure 1: Train-track-bridge model.

(2) shows that the calculation of force of wheel-rail is related to the structural response of the wheel; therefore, iteration is needed in the implicit algorithm or using the explicit algorithm [37].

\section{Karhunen-Loéve Expansion}

3.1. Theory. It is assumed that $u(x, \theta)$ is a real-valued stochastic process, and $\bar{u}(x)$ is the mean value of $u(x, \theta)$, and $C\left(x_{1}, x_{2}\right)$ is a covariance function. According to Mercer's theorem, $C\left(x_{1}, x_{2}\right)$ is bounded, symmetric, and positive and can be expanded as follows:

$$
C\left(x_{1}, x_{2}\right)=\sum_{n=1}^{\infty} \lambda_{n} \varphi_{n}\left(x_{1}\right) \varphi_{n}\left(x_{2}\right),
$$

where $\lambda_{n}$ and $\varphi_{n}(x)$ are the eigenvalues and eigenfunctions of $C\left(x_{1}, x_{2}\right)$, respectively. Equation (7) can be solved using the following equation:

$$
\int_{D} C\left(x_{1}, x_{2}\right) \varphi_{n}\left(x_{1}\right) \mathrm{d} x=\lambda_{n} \varphi_{n}\left(x_{2}\right) .
$$

The eigenfunctions $\varphi_{n}(x)$ satisfy orthogonal property, and it can be written as follows:

$$
\int_{D} \varphi_{n}\left(x_{1}\right) \varphi_{m}\left(x_{2}\right) \mathrm{d} x=\delta_{n m},
$$

where $\delta_{n m}$ is the Kronecker delta. The stochastic process $u(x, \theta)$ can be expressed as follows:

$$
u(x, \theta)=\bar{u}(x, \theta)+\sum_{n=1}^{\infty} \xi_{n}(\theta) \sqrt{\lambda_{n}} \varphi_{n}(x) .
$$

This is the KLE, where $\xi_{n}(\theta)$ is a set of uncorrelated random variables, which can also be written as follows:

$$
\xi_{n}(\theta)=\frac{1}{\sqrt{\lambda_{n}}} \int_{D} \tilde{u}(x, \theta) \varphi_{n}(x) \mathrm{d} x .
$$

When $u(x, \theta)$ belongs to the Gaussian stochastic process, $\xi_{n}(\theta)$ will be a group of uncorrelated variables, obeying the standard normal distribution.

In application, the first $M$ terms in equation (10) should be truncated, which can be expressed as follows:

$$
u(x, \theta)=\bar{u}(x, \theta)+\sum_{n=1}^{M} \xi_{n}(\theta) \sqrt{\lambda_{n}} \varphi_{n}(x) .
$$

The accuracy of representation of stochastic field depends on $M$.

3.2. Numerical Expression. It is assumed that $\mathbf{r}(x, \theta)$ is a spatial one-dimensional stochastic process; its length is $L$. Isometric discretization of stochastic processes is $\Delta x$, and the number of discrete points is $n=L / \Delta x+1$. After enough stochastic samples were obtained, the stochastic process $\mathbf{r}(x, \theta)$ can be represented as follows.

There is a sample set of stochastic $\mathbf{R}(x, \theta)=\left\{\mathbf{r}_{1}(x, \theta)\right.$; $\left.\mathbf{r}_{2}(x, \theta), \ldots, \mathbf{r}_{m}(x, \theta)\right\}$, where $m$ is the number of samples and $\mathbf{r}_{i}(x, \theta)$ is one of the samples with order $1 \times n$. The covariance matrix $\Gamma_{\mathbf{R}, \mathbf{R}}$ with order $n \times n$ can be obtained. Then, the corresponding eigenvalues and eigenfunctions of stochastic process can be calculated using the following equation:

$$
\left(\Gamma_{\mathbf{R}, \mathbf{R}} \Delta x\right) \varphi_{i}-\lambda_{i} \varphi_{i}=0,
$$

where $\lambda_{n}$ and $\varphi_{n}(x)$ are the eigenvalues and eigenfunctions of KLE, respectively, and $\varphi_{n}(x)$ should be normalized.

After the truncation at the $M^{\text {th }}$ order, the stochastic process can be represented by the KLE, which is denoted as follows:

$$
\mathbf{r}(x, \theta)=\bar{r}(x, \theta)+\sum_{n=1}^{M} \xi_{n}(\theta) \sqrt{\lambda_{n}} \varphi_{n}(x),
$$

where $\bar{r}(x, \theta)$ is the expectation of $\mathbf{R}(x, \theta)$. 


\section{Point Estimation Method}

It is assumed that $p(x)$ is a probability density function (PDF) of a continued random variable $Y$, and $Y=g(X)$. The expectation of random variable can be calculated using the following equation:

$$
\mu=E[g(\mathbf{X})]=\int_{-\infty}^{\infty} G(\mathbf{X}) p(\mathbf{X}) d \mathbf{X}
$$

The variance of $Y$ can be calculated using the following formula:

$$
\sigma^{2}=\int_{-\infty}^{\infty}[g(\mathbf{X})-\mu]^{2} p(\mathbf{X}) \mathrm{d} \mathbf{X}
$$

When the function contains multiple random variables, it may be difficult to calculate the moments such as the central moments of $Y=G(X)$. According to the dimension reduction method for multiple variables function proposed in [38], a $n$-dimensional variable function $g(X)$ can be approximated using several $s$-dimensional variable function $g^{s}(X)$, which can be written as follows:

$$
g(\mathbf{X}) \cong g^{s}(\mathbf{X})=\sum_{i=0}^{s}(-1)^{i} C_{n-s+i-1}^{i} \sum_{k_{1}<\cdots<k_{s-i}} y_{s-i},
$$

with $y_{s-i}=g\left(c_{1}, \ldots, c_{k_{1}-1}, x_{k_{1}}, c_{k_{4}+} 1, \ldots, c_{k_{c-1}-1}, x_{k_{c-i}}, c_{k_{c-1}+1}\right.$, $\left.\ldots, c_{n}\right)$, where $s<n$ and $c=\left[c_{1}, c_{2}, \ldots, c_{n}\right]$ is the reference point.

When $s=1$, the function is a one-variable dimensional reduction. Equation (17) can be converted into the following equation:

$$
g(\mathbf{X}) \cong g^{1}(\mathbf{X})=\sum_{i=1}^{n} g_{i}\left(X_{i}\right)-(n-1) g(c),
$$

with $g_{i}\left(X_{i}\right)=g\left(c_{1}, \ldots, c_{i-1}, X_{i}, c_{i+1}, \ldots, c_{n}\right)$.

By substituting equation (18) into equations (15) and (16), the expectation and variance of $Y$ can be approximately expressed as follows:

$$
\begin{gathered}
\mu=E\left[\sum_{i=1}^{n} g_{i}\left(X_{i}\right)-(n-1) g(c)\right] \cong \sum_{i=1}^{n} E\left[g_{i}\left(X_{i}\right)\right]-(n-1) g(c) \\
\sigma^{2} \cong E\left[\sum_{i=1}^{n}\left(g_{i}-\mu\right)^{2}-(n-1)[g(c)-\mu]^{2}\right] \\
=\sum_{i=1}^{n} E\left\{\left[g_{i}\left(X_{i}\right)-\mu\right]^{2}\right\}-(n-1)[g(c)-\mu]^{2}
\end{gathered}
$$

When the random variable in function $g_{i}\left(\mathbf{X}_{i}\right)$ obeys the standard Gaussian distribution, $E\left[g_{i}\left(X_{i}\right)\right]$ and $E\left[\left(g_{i}\left(X_{i}\right)-\mu\right)^{2}\right]$ can be calculated approximately using the Gaussian-Hermite integration function, which can be expressed as follows:

$$
\begin{gathered}
E\left[g_{i}\left(X_{i}\right)\right]=\sum_{l=1}^{r} \frac{w_{\mathrm{GH}, l}}{\sqrt{\pi}} g_{i}\left(\sqrt{2} x_{\mathrm{GH}, l}\right), \\
E\left[\left(g\left(X_{i}\right)-\mu\right)^{2}\right]=\sum_{l=1}^{r} \frac{w_{\mathrm{GH}, l}}{\sqrt{\pi}}\left[g_{i}\left(\sqrt{2} x_{\mathrm{GH}, l}\right)-\mu\right]^{2},
\end{gathered}
$$

where $r$ is the number of estimating points of Gaussian-Hermite integration and $x_{\mathrm{GH}, l}$ and $w_{\mathrm{GH}, l}$ are the abscissas and weights for Gaussian-Hermite integration, respectively; their detailed values with $r=3$ are shown in Table 1.

\section{Stochastic Dynamic Method for TTBS}

Samples of track irregularity can be obtained from the actual railway line measurement or converted into spatial samples using power spectral density (PSD). After obtaining multiple spatial samples for irregularity, the eigenvalues and eigenfunctions expressed by the KLE of track irregularity can be derived. Assuming track irregularity $\widetilde{z}_{R}(x, \theta)$ as a Gaussian random process, its KLE can be expressed as follows:

$$
z(x)=\bar{z}(x)+\sum_{k=1}^{m} \sqrt{\lambda_{k}} \xi_{k} \varphi_{k}(x)
$$

where $\bar{z}(x, \theta)$ is the mean value of track irregularity and $\xi_{k}$ is a set of distributed random variables independent of each other. Track irregularity is a stochastic process with zero mean value; thus, $\bar{z}(x, \theta)=0$. Equation (22) can be simplified as follows:

$$
z(x)=\bar{z}(x)+\sum_{k=1}^{m} \sqrt{\lambda_{k}} \xi_{k} \varphi_{k}(x) .
$$

After obtaining an irregularity sample, it can be calculated as the irregularity amplitude in the model, i.e., the irregularity acts on the wheel-rail force of the train-bridge system as an external random excitation, and the corresponding response of the TTBS can be obtained. Therefore, the entire TTBS is a stochastic system with $m$ random variables. According to the characteristics of equation (23), when the PEM is used for stochastic response calculation, zero can be selected as the reference point. In the calculation of stochastic system response through point estimation, the track irregularity sample corresponding to the $l^{\text {th }}$ Gaussian point of $k^{\text {th }}$ random variable can be expressed as follows:

$$
\tilde{z}_{k, l}(x)=\sqrt{2} x_{\mathrm{GH}, l} \sqrt{\lambda_{k}} \varphi_{k}(x) .
$$

Taking the displacement response of time $t$ at some point of the bridge for instance, the corresponding response of the system $R(k, l, t)$ can be obtained after calculating the track irregularity samples corresponding to each Gaussian point of each stochastic variable as the track irregularity samples of 
TABLE 1: Abscissas and weights for Gaussian-Hermite integration with $r=3$ [39].

\begin{tabular}{lccc}
\hline Point & 1 & 2 & 3 \\
\hline$x_{\mathrm{GH}, l}$ & -1.22474 & 0 & 1.22474 \\
$w_{\mathrm{GH}, l}$ & 0.29541 & 1.18164 & 0.29541 \\
\hline
\end{tabular}

the system. The corresponding response of zero-irregularity sample is $R_{0}(t)$. The expectation and variance of response can be obtained by substituting all $R(k, l, t)$ and $R_{0}$ into equations (19) and (20):

$$
\begin{aligned}
\text { Mean } \cdot(t) \approx & \sum_{k=1}^{m} \sum_{l=1}^{r} \frac{w_{\mathrm{GH}, l}}{\sqrt{\pi}} R(k, l, t)-(m-1) R_{0}(t), \\
\operatorname{Var} \cdot(t)= & \sum_{n=1}^{m} \sum_{l=1}^{r} \frac{w_{\mathrm{GH}, l}}{\sqrt{\pi}}[R(k, l, t)-\operatorname{Mean} \cdot(t)]^{2}-(m-1) \\
& \cdot\left[R_{0}(t)-\text { Mean } \cdot(t)\right]^{2} .
\end{aligned}
$$

Except the nonzero Gaussian point of $k^{\text {th }}$ stochastic variable, other track irregularity samples are zero-amplitude irregularity, which is $R(k,(r+1) / 2, t)=R_{0}(t)$. Therefore, there is no need to repeat the calculation in the program. The standard deviation value of response can be calculated using the following equation:

$$
\operatorname{Std} . \mathrm{D}(t)=\sqrt{\operatorname{Var} \cdot(t)} .
$$

\section{Numerical Simulation}

Track irregularity can be measured using the railway line [9] and can also be obtained by converting the existing PSD function into spatial samples. Power spectrum simulation is used to obtain irregularity samples, where German lowinterference PSD is used [12]. 10,000 irregularity samples with 1,000 meters are obtained using the trigonometric series method [8] and utilized as the initial sample. The results indicate that KLE truncation number 138 can obtain more than $98 \%$ of the probability information; therefore, the number of random variables is considered as 138 .

\subsection{Comparison and Validation}

Case 1 (a simple model). The nonlinear wheel-rail contact model may cause some trouble for the calculation of the stochastic TTBS; for example, the Hertz spring should be linearized in stochastic analysis [40]; besides, in the case of extreme track irregularity condition, the wheelset may jump, i.e., then there is no contact between the wheel and rail. To evaluate the applicability of the proposed method in the random TTBS with random track irregularity, a simple model is established, as shown in Figure 2. This model contains three DOFs, carbody-bogie system, wheel, and track. Besides gravity, the simple system is also excited by a random irregularity. When running to the third second, a $5 \mathrm{~mm}$ additional irregularity is suddenly added to force the

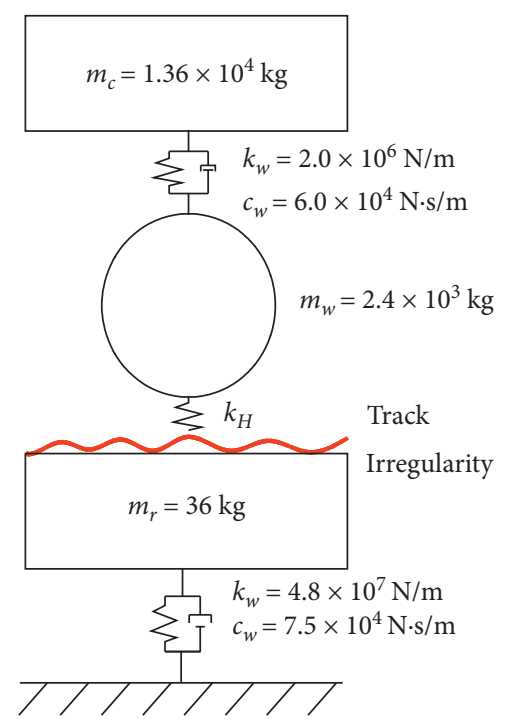

Figure 2: Simple model of the train-track system.

wheel to jump to analyze the calculation accuracy of the proposed method after the wheel jump. The MCS method is used to compare and verify the accuracy of KLE-PEM. The mean and standard deviation values of displacement response of the wheel calculated using two methods are shown in Figure 3; it is observed that the dynamic response of the system enters the stable stage in two seconds. After being subjected to a sudden track irregularity excitation at the $3^{\text {rd }}$ second, the wheel jumps from the track and then falls back to the track and returns to stability in about $0.2 \mathrm{~s}$. In the entire process, the mean and standard deviation values of response calculated using the KLE-PEM are very close to those obtained by MCS, i.e., the proposed method can accurately calculate random responses with nonlinear wheel-rail contact even in the case of a jump, and it can be used to study extreme irregularities such as earthquake, settlement, creep, and rail damage in the future.

Case 2 (comparison with MCS). The comparison results obtained with MCS are used to validate the accuracy of KLEPEM in the nonlinear stochastic TTBS. In this case, only one carriage and one-span bridge are evaluated to save time, and the subgrade section is rigid. The calculation load of the TTBS for MCS is 5000. The number of KLE items after truncation is 138, and when using three nodes of Gaussian quadrature, the calculation load of the TTBS using KLEPEM is $138 \times 2+1=277$. Wheel-rail contact force is calculated according to a nonlinear Hertz spring. The train parameters [41] are shown in Table 2, and the parameters of the bridge and rail system are shown in Table 3.

Figure 4 shows the comparison of mean and standard deviation values and vertical displacement of rail fastener in the middle span calculated using KLE-PEM and MCS. It can be concluded that for the stochastic dynamic response of the track-bridge system, the results of KLE-PEM are almost consistent with the results of MCS. Figure 5 shows a comparison of the time-histories response of mean and standard deviation of the first wheel-rail force passing 


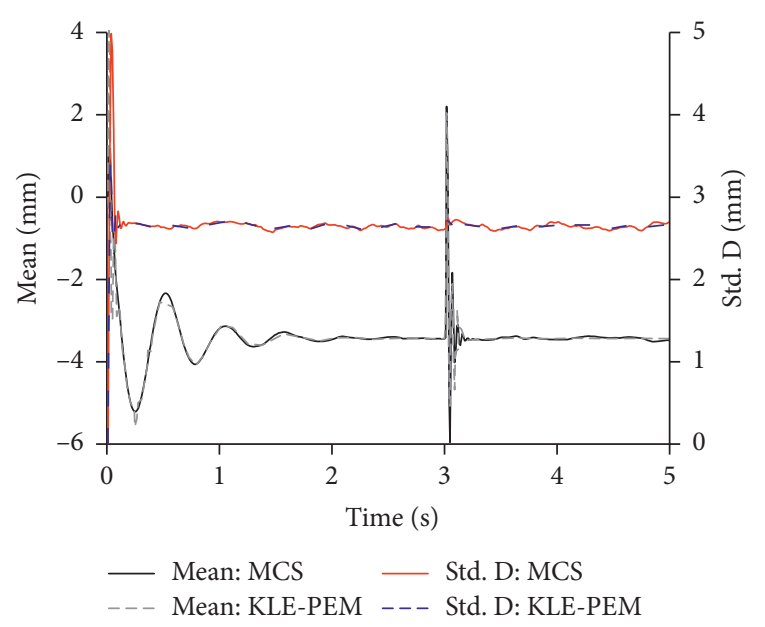

Figure 3: Comparison of displacement of the wheel.

TABLe 2: Properties of train.

\begin{tabular}{lcc}
\hline Notation & Unit & Trailer car/motor car \\
\hline$m_{c}$ & $\mathrm{~kg}$ & $4.40 \times 10^{4} / 4.80 \times 10^{4}$ \\
$m_{t}$ & $\mathrm{~kg}$ & $2.40 \times 10^{3} / 3.20 \times 10^{3}$ \\
$m_{w}$ & $\mathrm{~kg}$ & $2.40 \times 10^{3} / 2.40 \times 10^{3}$ \\
$J_{c}$ & $\mathrm{~kg} \cdot \mathrm{m}^{2}$ & $2.70 \times 10^{6} / 2.70 \times 10^{6}$ \\
$J_{t}$ & $\mathrm{~kg} \cdot \mathrm{m}^{2}$ & $2.20 \times 10^{3} / 7.20 \times 10^{3}$ \\
$K_{1 z}$ & $\mathrm{~N} / \mathrm{m}$ & $0.70 \times 10^{6} / 1.00 \times 10^{6}$ \\
$K_{2 z}$ & $\mathrm{~N} / \mathrm{m}$ & $3.00 \times 10^{5} / 4.00 \times 10^{5}$ \\
$C_{1 z}$ & $\mathrm{~N} \cdot \mathrm{s} / \mathrm{m}$ & $4.00 \times 10^{4} / 3.00 \times 10^{4}$ \\
$C_{2 z}$ & $\mathrm{~N} \cdot \mathrm{s} / \mathrm{m}$ & $5.00 \times 10^{4} / 4.00 \times 10^{4}$ \\
$L_{c}$ & $\mathrm{~m}$ & $8.6875 / 8.6785$ \\
$L_{t}$ & $\mathrm{~m}$ & $1.25 / 1.25$ \\
$L_{w w}$ & $\mathrm{~m}$ & $4.90 / 4.90$ \\
\hline
\end{tabular}

through the bridge. It can also be concluded that the results calculated using KLE-PEM are almost consistent with the results obtained using MCS.

6.2. Comparison between Linear and Nonlinear Simulation. A standard simply supported 7-span box girder with $32 \mathrm{~m}$ length per span is modeled to evaluate the effect of random track irregularity on the TTBS, and the difference between linear and nonlinear Hertz contacts is evaluated. A rigid subgrade is used for the simulation out of the bridge part. The treatment of boundary conditions between adjacent spans is shown in Figure 1. The train uses four carriages ICE3 train, and there are two motor cars at the head and tail of the train. To ensure the dynamic response stability before the train entering the bridge, the train starts running from $300 \mathrm{~m}$ away from the left side of the first span bridge. The operation speed of the train is $250 \mathrm{~km} / \mathrm{h}$. The TTBS considering linear Hertz and nonlinear Hertz is established.

Figure 6 shows the mean and standard deviation of midspan displacement response of fourth bridge span under the nonlinear and linear wheel-rail relationship, where the horizontal ordinate represents the time for the first wheelset entering the fourth bridge span. It can be concluded that the bridge dynamic responses under the two wheel-rail force models are similar. In addition, in the perspective of timehistories response trend, the mean value of time-histories response of the bridge has two peaks and three troughs. However, the standard deviation has only two peaks before and after, and the time of the first peak is inconsistent with the time of the first trough of the mean value.

The mean and standard deviation of time-histories response of vertical acceleration at the center of gravity of the first carbody are shown in Figure 7, where the horizontal ordinate is the time taken for the first wheelset entering the first bridge span. It shows that the standard deviations of train acceleration obtained by the two wheel-rail force models are inconsistent. The standard deviation obtained by linear wheel-rail relationship is less than that obtained by nonlinear wheel-rail relationship in a relatively large time. In the entire process, the values of standard deviation obtained from the linear wheel-rail relationship are all around $6.7 \times 10^{-2} \mathrm{~m} / \mathrm{s}^{2}$, whereas the standard deviations obtained from the nonlinear relationship are relatively large at the initial stage of entering the bridge and then gradually decreases to $6.8 \times 10^{-2} \mathrm{~m} / \mathrm{s}^{2}$.

The mean and standard deviation of the time-histories response of vertical wheel-rail contact force of the first wheelset are shown in Figure 8, where the horizontal ordinate is the time taken for the first wheelset entering the first bridge span. Because the subgrade section adopts rigid subgrade, there will be greater contact force at the moment when the wheelsets drive into and out of the bridge. Therefore, the wheel-rail force of the second span to the sixth span of the wheel set is selected for analysis; it can be seen that the mean values of contact force calculated by the nonlinear and linear model both oscillate about $20 \mathrm{kN}$ along the axle load, and the magnitude of the contact force obtained from the nonlinear model is larger than that of the linear model. Regarding the standard deviations of wheelrail force, it shows a significant difference between the two models at the initial stage when the train enters the bridge. Then, the difference gradually decreases, both of which oscillate between $2.5 \mathrm{kN}$ and $4 \mathrm{kN}$. The standard deviations obtained from the linear wheel-rail relationship are less than that obtained from the nonlinear wheel-rail relationship in a relatively long time.

6.3. Applied for Response Prediction. When it is necessary to obtain the response value of the bridge, the most accurate method is to install sensors in the field for actual measurement, but this method is very time-consuming and inconvenient. Therefore, the numerical simulation method is usually used to obtain the bridge response. However, if the deterministic analysis is used, the result will be different from the actual value because there are various randomness in the TTBS, such as track irregularity, train load, and so on. The results of probability range can be obtained by using the proposed stochastic analysis method. The accuracy of the probability distribution range obtained by KLE-PEM can be verified by comparing the test results in Ref. [42]. In this case, besides the stochastic track irregularity, there is also the 
TABle 3: Properties of the track-bridge.

\begin{tabular}{|c|c|c|c|}
\hline Notation & Unit & Item & Value \\
\hline$E_{r}$ & $\mathrm{~N} / \mathrm{m}^{2}$ & Young's modulus of rail & $2.06 \times 10^{11}$ \\
\hline$v_{r}$ & - & Poisson's ratio of rail & 0.3 \\
\hline$I_{r}$ & $\mathrm{~m}^{4}$ & Mass moment of inertia of rail cross section & $2 \times 3.217 \times 10^{-5}$ \\
\hline $\bar{m}_{r}$ & $\mathrm{~kg} / \mathrm{m}$ & Mass per unit length of rail & $2 \times 60.64$ \\
\hline$E_{s}$ & $\mathrm{~N} / \mathrm{m}^{2}$ & Young's modulus of slab & $3.6 \times 10^{10}$ \\
\hline$v_{s}$ & - & Poisson's ratio of slab & 0.2 \\
\hline$I_{s}$ & $\mathrm{~m}^{4}$ & Mass moment of inertia of slab cross section & $1.6 \times 10^{-3}$ \\
\hline $\bar{m}_{s}$ & $\mathrm{~kg} / \mathrm{m}$ & Mass per unit length of slab & $1.2 \times 10^{3}$ \\
\hline$k_{r s}^{s}$ & $\mathrm{~N} / \mathrm{m}$ & Spring stiffness of fastener & $2 \times 5.0 \times 10^{7}$ \\
\hline$c_{r s}$ & $\mathrm{~N} \cdot \mathrm{s} / \mathrm{m}$ & Damping coefficient of fastener & $2 \times 3.625 \times 10^{4}$ \\
\hline $\bar{k}_{s b}$ & $\mathrm{~N} / \mathrm{m}^{2}$ & Spring stiffness of CA layer per unit length & $2 \times 1.5 \times 10^{9}$ \\
\hline $\bar{c}_{s b}$ & $\mathrm{~N} \cdot \mathrm{s} / \mathrm{m}^{2}$ & Damping coefficient of CA layer per unit length & $2 \times 8.3 \times 10^{4}$ \\
\hline$E_{b}$ & $\mathrm{~N} / \mathrm{m}^{2}$ & Young's modulus of girder & $3.45 \times 10^{10}$ \\
\hline$v_{b}$ & - & Poisson's ratio of girder & 0.2 \\
\hline$I_{b}$ & $\mathrm{~m}^{4}$ & Mass moment of inertia of girder cross section & 12.744 \\
\hline $\bar{m}_{b}$ & $\mathrm{~kg} / \mathrm{m}$ & Mass per unit length of bridge girder & $2.972 \times 10^{4}$ \\
\hline$\zeta_{b}$ & - & Damping ratio of girder & 0.05 \\
\hline
\end{tabular}

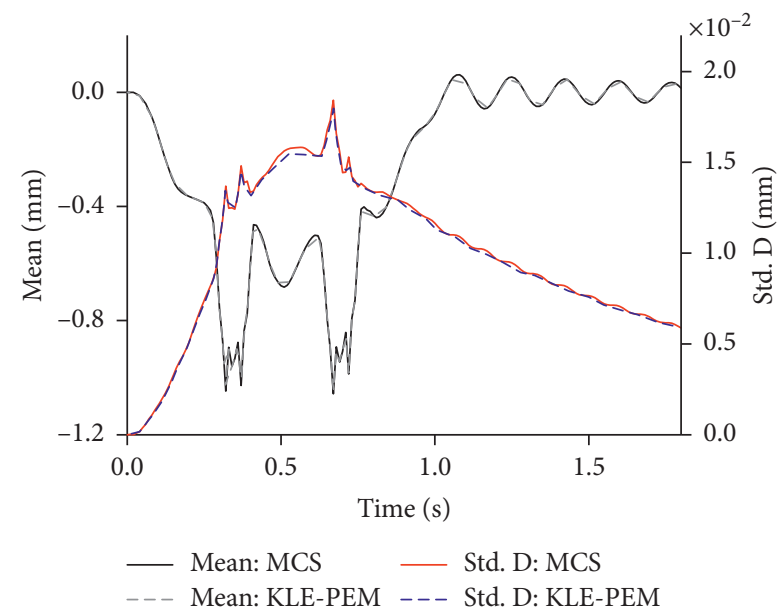

Figure 4: Comparison of displacement of fastener at midspan.

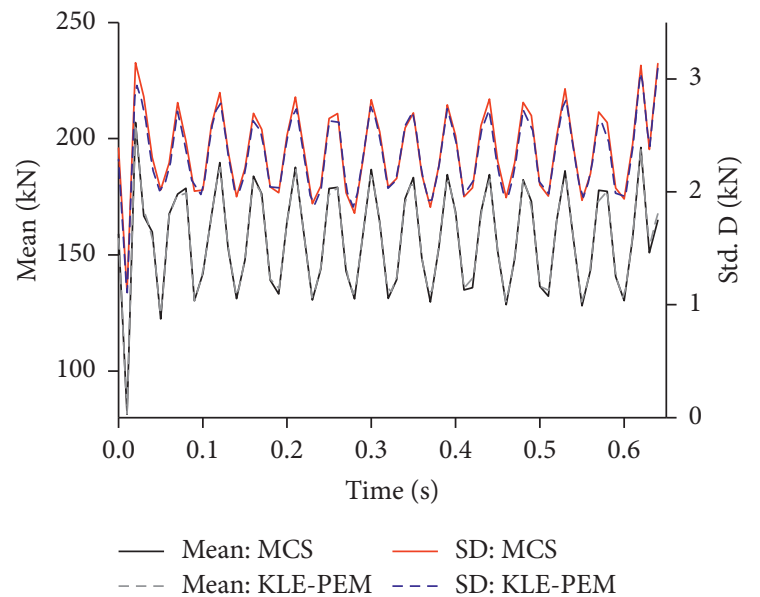

FIGURE 5: Comparison of wheel-rail contact force of first wheelset (double side).

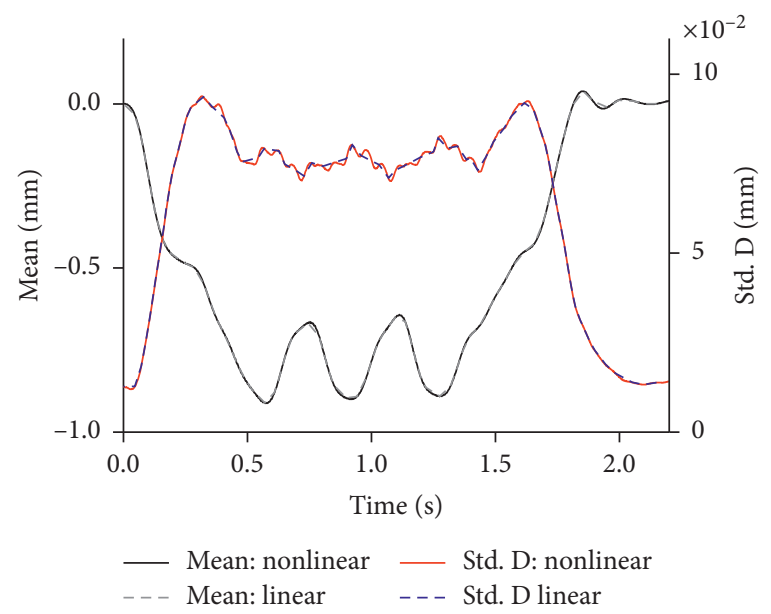

Figure 6: Vertical displacement of midspan of fourth span.

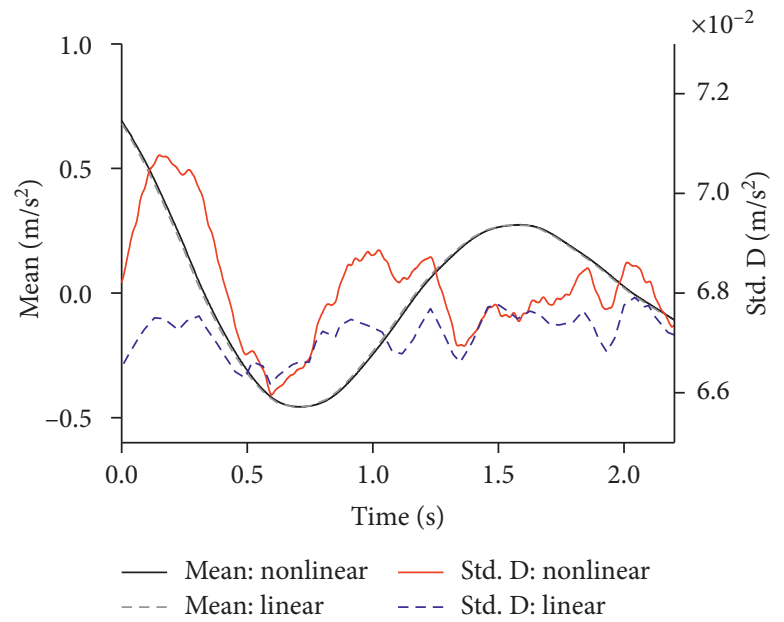

FIgURE 7: Vertical acceleration at the center of gravity of first carbody. 


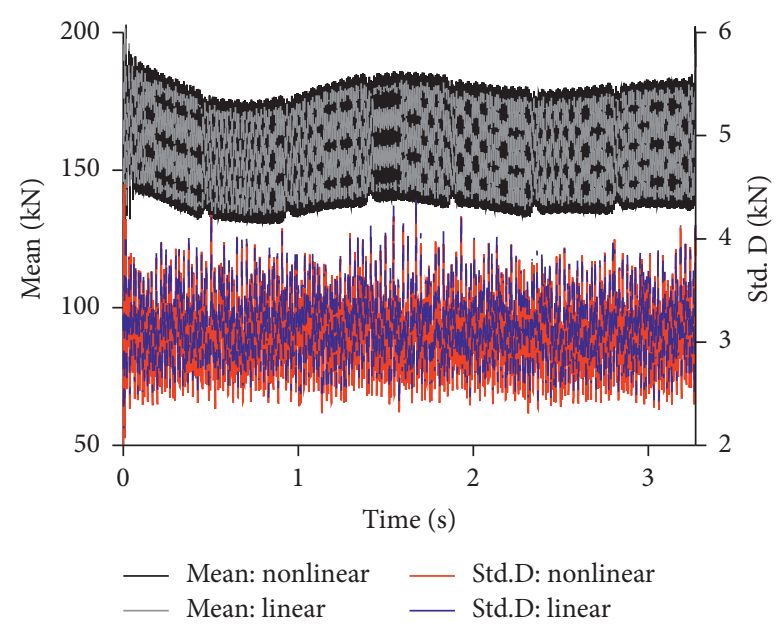

Figure 8: Vertical contact force of first wheelset (double side).

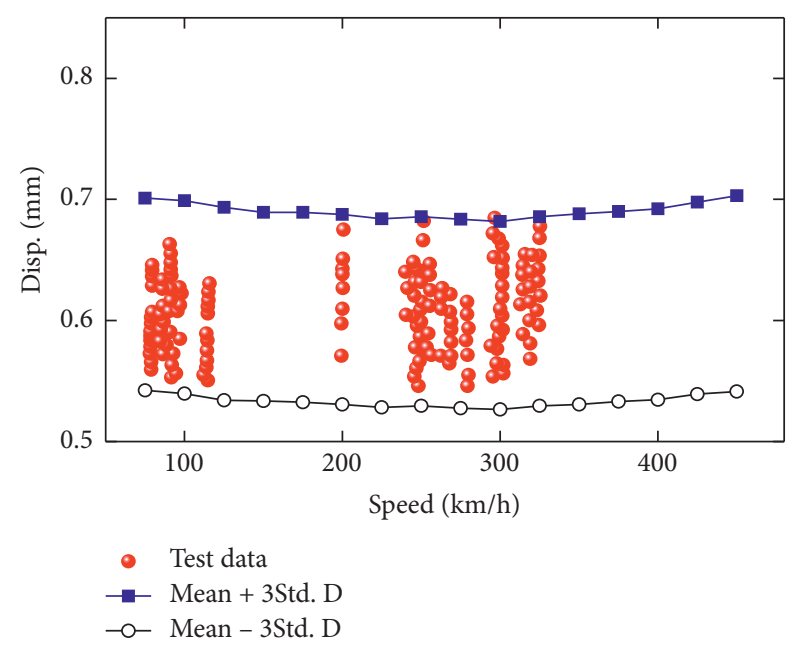

Figure 9: Calculation results and test data.

random axle load, in which the train carbody mass is a normal distribution random variable with a coefficient of variation of 0.05 . It was proved that the PEM can calculate the response of the TTBS with uncertain parameter precisely [24]; therefore, only one independent random variable needs to be added in KLE-PEM. (Mean \pm 3 Std. D) are used to guarantee the probability range of results; the calculation results and the test data of maximum values of vertical displacement at bridge midspan are shown in Figure 9. It can be found that the test results are basically within the calculated probability distribution range, which means that the proposed method can be used to effectively predict the distribution range of response results.

\section{Conclusions}

In this study, a stochastic analysis method KLE-PEM is introduced to analyze the random train-track-bridge dynamic model, and the difference between the nonlinear and linear Hertz contact model is discussed, and the proposed method compared with the actual measurement results mainly concluded as the following results:

(1) The proposed method can accurately calculate the random response after the wheel jumps from the extreme track irregularity.

(2) The mean and standard deviation of the stochastic dynamic system through KLE-PEM and MCS are approximate. Meanwhile, under the same accuracy, KLE-PEM is an order of magnitude higher than MCS.

(3) In the TTBS, nonlinear and linear wheel-rail contact models are compared. It can be concluded that there is almost no difference in the effect of these two models on the bridge response. The effects on train response are different at the initial stage when the train enters a bridge, but with the train operation, the difference decreases gradually.

(4) KLE-PEM can be used to effectively predict the distribution range of response.

\section{Data Availability}

The data used to support the findings of the study are included within the article.

\section{Conflicts of Interest}

The authors declare that they have no conflicts of interest.

\section{Acknowledgments}

The work described in this paper was supported by grants from the National Natural Science Foundation of China (grant nos. U1934207, 51778630, and 11972379) and the Fundamental Research Funds for the Central Universities of Central South University (grant no. 2020zzts148).

\section{References}

[1] Y. Zhang, L. Jiang, W. Zhou, Y. Feng, Z. Tan, and X. Chai, "Study of bridge-subgrade longitudinal constraint range for high-speed railway simply-supported beam bridge with CRTSII ballastless track under earthquake excitation," Construction and Building Materials, vol. 241, Article ID 118026, 2020.

[2] L. Jiang, Y. Zhang, Y. Feng, W. Zhou, and Z. Tan, "Simplified calculation modeling method of multi-span bridges on highspeed railways under earthquake condition," Bulletin of Earthquake Engineering, vol. 18, no. 5, pp. 2303-2328, 2020.

[3] A. Paixão, E. Fortunato, and R. Calçada, "The effect of differential settlements on the dynamic response of the traintrack system: a numerical study," Engineering Structures, vol. 9, 2015.

[4] Y.-S. Wu and Y.-B. Yang, "Steady-state response and riding comfort of trains moving over a series of simply supported bridges," Engineering Structures, vol. 25, no. 2, pp. 251-265, 2003.

[5] J. Yu, L. Jiang, W. Zhou, X. Liu, Z. Lai, and Y. Feng, "Study on the dynamic response correction factor of a coupled highspeed train-track-bridge system under near-fault 
earthquakes," Mechanics Based Design of Structures and Machines, pp. 1-19, 2020.

[6] L. Jiang, Y. Zhang, Y. Feng, W. Zhou, and Z. Tan, "Dynamic response analysis of a simply supported double-beam system under successive moving loads," Applied Sciences, vol. 9, no. 10, p. 2162, 2019.

[7] Z. Lai, L. Jiang, and W. Zhou, "An analytical study on dynamic response of multiple simply supported beam system subjected to moving loads," Shock and Vibration, vol. 2018, pp. 1-14, 2018.

[8] Z. Lai, L. Jiang, X. Liu, Y. Zhang, and W. Zhou, "Analytical investigation on the geometry of longitudinal continuous track in high-speed rail corresponding to lateral bridge deformation," Construction and Building Materials, Article ID 121064, 2020.

[9] M. Zhu, X. Cheng, L. Miao, and X. Sun, "Random field modeling of track irregularity of Beijing-Guangzhou highspeed railway with Karhunen-Loève expansion," International Journal of Distributed Sensor Networks, vol. 11, no. 6, Article ID 521437, 2015.

[10] J. M. Rocha, A. A. Henriques, and R. Calçada, "Probabilistic safety assessment of a short span high-speed railway bridge," Engineering Structures, vol. 71, pp. 99-111, 2014.

[11] S. Zhu, C. Cai, and W. Zhai, "Interface damage assessment of railway slab track based on reliability techniques and vehicletrack interactions," Journal of Transportation Engineering, vol. 142, Article ID 04016041, 2016.

[12] X. Li, Y. Zhu, and Z. Jin, "Nonstationary random vibration performance of train-bridge coupling system with vertical track irregularity," Shock and Vibration, vol. 2016, Article ID 1450895, 19 pages, 2016.

[13] Z.-P. Zeng, Z.-W. Yu, Y.-G. Zhao, W.-T. Xu, L.-K. Chen, and P. Lou, "Numerical simulation of vertical random vibration of train-slab track-bridge interaction system by PEM," Shock and Vibration, vol. 2014, pp. 1-21, 2014

[14] F. Lu, J. H. Lin, D. Kennedy, and F. W. Williams, "An algorithm to study non-stationary random vibrations of vehicle-bridge systems," Computers \& Structures, vol. 87, no. 34, pp. 177-185, 2009.

[15] J. Zhang, Y. Zhao, Y.-H. Zhang et al., "Non-stationary random vibration of a coupled vehicle-slab track system using a parallel algorithm based on the pseudo excitation method," Proceedings of the Institution of Mechanical Engineers, Part F: Journal of Rail and Rapid Transit, vol. 227, no. 3, pp. 203-216, 2013.

[16] Y. Zhu, X. Li, and Z. Jin, "Three-dimensional random vibrations of a high-speed-train-bridge time-varying system with track irregularities," Proceedings of the Institution of Mechanical Engineers, Part F: Journal of Rail and Rapid Transit, vol. 230, no. 8, pp. 1851-1876, 2016.

[17] Z.-w. Yu, J.-f. Mao, F.-q. Guo, and W. Guo, "Non-stationary random vibration analysis of a $3 \mathrm{D}$ train-bridge system using the probability density evolution method," Journal of Sound and Vibration, vol. 366, pp. 173-189, 2016.

[18] L. Xu, W. Zhai, and J. Gao, "A probabilistic model for track random irregularities in vehicle/track coupled dynamics," Applied Mathematical Modelling, vol. 51, pp. 145-158, 2017.

[19] L. Xu, W. Zhai, and Z. Li, "A coupled model for train-trackbridge stochastic analysis with consideration of spatial variation and temporal evolution," Applied Mathematical Modelling, vol. 63, pp. 709-731, 2018.

[20] L. Xu and W. Zhai, "Stochastic analysis model for vehicletrack coupled systems subject to earthquakes and track random irregularities," Journal of Sound and Vibration, vol. 407, pp. 209-225, 2017.

[21] L. Xu and W. Zhai, "A novel model for determining the amplitude-wavelength limits of track irregularities accompanied by a reliability assessment in railway vehicle-track dynamics," Mechanical Systems and Signal Processing, vol. 86, pp. 260-277, 2017.

[22] H.-P. Wan and Y.-Q. Ni, "An efficient approach for dynamic global sensitivity analysis of stochastic train-track-bridge system," Mechanical Systems and Signal Processing, vol. 117, pp. 843-861, 2019.

[23] S. Q. Wu and S. S. Law, "Vehicle axle load identification on bridge deck with irregular road surface profile," Engineering Structures, vol. 33, no. 2, pp. 591-601, 2011.

[24] L. Jiang, X. Liu, P. Xiang, and W. Zhou, “Train-bridge system dynamics analysis with uncertain parameters based on new point estimate method," Engineering Structures, vol. 199, Article ID 109454, 2019.

[25] E. Rosenblueth, "Point estimates for probability moments," Proceedings of the National Academy of Sciences, vol. 72, no. 10, pp. 3812-3814, 1975.

[26] X. Liu, L. Jiang, Z. Lai, P. Xiang, and Y. Chen, "Sensitivity and dynamic analysis of train-bridge coupled system with multiple random factors," Engineering Structures, vol. 221, Article ID 111083, 2020.

[27] X. Liu, P. Xiang, L. Jiang, Z. Lai, T. Zhou, and Y. Chen, "Stochastic analysis of train-bridge system using the Karhunen-Loéve expansion and the point estimate method," International Journal of Structural Stability and Dynamics, vol. 20, no. 2, Article ID 2050025, 2020.

[28] W. Zhai, Z. Han, Z. Chen, L. Ling, and S. Zhu, "Train-trackbridge dynamic interaction: a state-of-the-art review," Vehicle System Dynamics, vol. 57, no. 7, pp. 984-1027, 2019.

[29] Z. Lai, X. Kang, L. Jiang et al., "Earthquake influence on the rail irregularity on high-speed railway bridge," Shock and Vibration, vol. 2020, Article ID 4315304, 16 pages, 2020.

[30] Y. Feng, L. Jiang, W. Zhou et al., "Experimental investigation on shear steel bars in CRTS II slab ballastless track under lowcyclic reciprocating load," Construction and Building Materials, vol. 255, Article ID 119425, 2020.

[31] Y. Feng, L. Jiang, W. Zhou, Z. Lai, and X. Chai, “An analytical solution to the mapping relationship between bridge structures vertical deformation and rail deformation of high-speed railway," Steel and Composite Structures, vol. 33, pp. 209-224, 2019.

[32] L. Jiang, L. Liu, W. Zhou, X. Liu, C. Liu, and P. Xiang, "Mapped relationships between pier settlement and rail deformation of bridges with CRTS III SBT," Steel and Composite Structures, vol. 36, pp. 481-492, 2020.

[33] C. Kang, S. Schneider, M. Wenner, and S. Marx, "Experimental investigation on rail fatigue resistance of track/ bridge interaction," Engineering Structures, vol. 216, p. $110747,2020$.

[34] C. Kang, M. Bode, M. Wenner, and S. Marx, "Experimental and numerical investigations of rail behaviour under compressive force on ballastless track systems," Engineering Structures, vol. 197, p. 109413, 2019.

[35] Z.-w. Yu and J.-f. Mao, "Probability analysis of train-trackbridge interactions using a random wheel/rail contact model," Engineering Structures, vol. 144, pp. 120-138, 2017.

[36] P. Lou and Q.-y. Zeng, "Formulation of equations of motion of finite element form for vehicle-track-bridge interaction system with two types of vehicle model," International Journal 
for Numerical Methods in Engineering, vol. 62, no. 3, pp. 435-474, 2005.

[37] W. Zhai, H. Xia, C. Cai et al., "High-speed train-track-bridge dynamic interactions - Part I: theoretical model and numerical simulation," International Journal of Rail Transportation, vol. 1, no. 1-2, pp. 3-24, 2013.

[38] S. Rahman and H. Xu, "A univariate dimension-reduction method for multi-dimensional integration in stochastic mechanics," Probabilistic Engineering Mechanics, vol. 19, no. 4, pp. 393-408, 2004.

[39] Y.-G. Zhao and T. Ono, "New point estimates for probability moments," Journal of Engineering Mechanics, vol. 126, no. 4, pp. 433-436, 2000.

[40] Z. Jin, S. Pei, X. Li, and S. Qiang, "Probabilistic evaluation approach for nonlinear vehicle-bridge dynamic performances," Journal of Sound and Vibration, vol. 339, pp. 143156, 2015.

[41] Z.-P. Zeng, X.-F. He, Y.-G. Zhao et al., "Random vibration analysis of train-slab track-bridge coupling system under earthquakes," Structural Engineering and Mechanics, vol. 54, no. 5, pp. 1017-1044, 2015.

[42] Z.-w. Yu and J.-f. Mao, "A stochastic dynamic model of traintrack-bridge coupled system based on probability density evolution method," Applied Mathematical Modelling, vol. 59, pp. 205-232, 2018. 